\title{
GUI DE BOURGOGNE, Chanson de geste du XIII siècle
}

\section{Philip E. Bennett}

\section{OpenEdition Journals}

Édition électronique

URL : https://journals.openedition.org/ccm/8811

DOI : $10.4000 / \mathrm{ccm} .8811$

ISSN : 2119-1026

\section{Éditeur}

Centre d'études supérieures de civilisation médiévale/Université de Poitiers

\section{Édition imprimée}

Date de publication : 1 décembre 2021

Pagination : $397-400$

ISBN : 978-2-490783-11-3

ISSN : 0007-9731

\section{Référence électronique}

Philip E. Bennett, « GUI DE Bourgogne, Chanson de geste du xII" siècle», Cahiers de civilisation médiévale [En ligne], 256 | 2021, mis en ligne le 01 décembre 2021, consulté le 03 juin 2022. URL : http:// journals.openedition.org/ccm/8811; DOI : https://doi.org/10.4000/ccm.8811

\section{(c) (i) $\odot$}

La revue Cahiers de civilisation médiévale est mise à disposition selon les termes de la Licence Creative Commons Attribution - Pas d'Utilisation Commerciale - Pas de Modification 4.0 International. 
Gui de Bourgogne, Chanson de geste du XIII siècle, Françoise E. Denis et William W. Kibler (éd.), Paris, Honoré Champion (Classiques français du Moyen Âge, 187), 2019.

Cette nouvelle édition de la chanson tardive de Gui de Bourgogne remplace de manière très opportune celle procurée au XIX ${ }^{\mathrm{e}} \mathrm{S}$. par François Guessard et Henri-Victor Michelant (Paris, Jannet [Les Anciens Poëtes de la France, 1], 1858). Cette édition souffre évidemment des faiblesses de celles de son époque : basée pour l'essentiel sur le manuscrit de Tours, Bibliothèque municipale, ms. 937, mais avec quelques leçons empruntées à celui de Londres, British Library, ms. Harley 527, sans que les éditeurs signalent clairement ces modifications de leur texte de base; la toilette du texte se conforme aux conventions de l'époque, 
qui sont maintenant tout à fait périmées. Le manuscrit de Londres et les deux fragments, Darmstadt, Hessische Landes- und Hochschulbibliotek, ms. 3306, et Sées, Archives diocésaines, ms. 19, ont été édités séparément, mais chaque fois sous une forme qui les rend difficilement accessibles. Le premier grand avantage, donc, de l'édition publiée par F. E. Denis et W. W. Kibler est qu'elle contient en regard le texte complet des deux manuscrits principaux et, en appendice, celui des deux fragments. Ainsi, chercheurs et étudiants ont maintenant à leur disposition, et sous un format très accessible, le corpus entier des témoins de cette chanson de geste dont l'intérêt a été souvent sous-estimé, parce que pour la plupart on a dû la lire dans l'édition de F. Guessard et H.-V. Michelant.

Avant d'aborder l'introduction, très détaillée (104 p.) et l'apparat critique, on peut utilement considérer la disposition dans le volume des textes édités. Le texte de Londres paraît à la fausse page et celui de Tours à la belle page. Ni l'un ni l'autre n'est présenté comme étant le texte de base, bien que celui de Tours ait eu ce privilège dans l'édition des Anciens Poëtes, parce que son copiste avait employé le kö̈né du français central continental, francien-picard-champenois, tandis que le texte de Londres est écrit en anglo-normand. Or, il y a tant de différences entre ces deux versions qu'il faut, sans doute, les considérer comme étant tous deux des révisions d'un original perdu. C'est effectivement ce que font F. E. Denis et W. W. Kibler. Non seulement les deux versions sont-elles de longueur très différente - Londres a 4598 vers, Tours 4296 - mais chacune offre des leçons inconnues de l'autre. Ainsi, lorsque l'un des manuscrits offre un ou plusieurs vers étrangers à l'autre, les éditeurs laissent un blanc dans le texte concerné, mais, si tel ou tel vers pourrait avoir un rapport même distant avec des vers dans l'autre manuscrit, on l'imprime en face à face pour éviter de créer « de trop nombreux vides dans les textes et [de prendre] trop d'espace » (p. 58). Pourtant, le choix de vers mis en regard et l'emplacement de blancs, indiquant l'absence de toute correspondance entre les textes, ne sont pas toujours évidents. Un passage, qui pose un nombre surélevé de problèmes, se trouve au milieu de la narration du combat singulier entre le héros, Gui, et Danemon, le fils du chef païen, Huidelon (v. 2857-2914 - L ; 2486-2538 - T). $\mathrm{Au}$ début du passage les deux premiers vers sont imprimés en face à face :

L : «Quant veit Danemon ke cil l'aveit feru,/Si en ot le quer dolent e irascu »; T: «Quant li rois Danemons sent que cil l'a feru,/Et Huidelons ses peres avoit le coup veü »; puis vient un blanc (L) vs « Bien set cil de la tor en ont grant joie eü » $(\mathrm{T})$; le prochain vers de chaque témoin est imprimé en face à face : $\mathrm{L}:$ « Pus en ot grant hunte del cop k'il a reçu »; T: «Plus li est de la honte que de l'ahan ne fu ».

En l'espèce, le deuxième vers de T n'a pas plus de correspondant dans L que le troisième; le lecteur doit, donc, se demander s'il n'aurait pas fallu laisser l'espace de deux blancs dans L. Plus loin dans ce même passage un autre problème se pose.

L : «Jamés ne verrez Charlon le veillard chanu, »; $\mathrm{T}$ : « Jamés ne reverrés Charlon le viel chanu, » suivi de trois blancs contre L : « Ne Rollant sun nevo n'Oliver sun dru;/Par Mahun mun Deu dunt ja tenc salu,/Jamés ne mangerai si vus avrai pendu »; le parallélisme reprend : $\mathrm{L}:$ « E tuz vos compaino[n]s de cele tur lasus. »; $\mathrm{T}:$ « $\mathrm{E}$ tous vos compaignons de ce palais volu »; enfin L a un blanc contre $\mathrm{T}$ : « Se je puis esploitier, seront ars ou pandu. »

Or, on remarque que le dernier vers cité de $\mathrm{T}$ (v. 2517) correspond grosso modo au v. 2885 de L, qui se trouve en regard du dernier blanc inséré dans le texte de $\mathrm{T}$. On se demande, donc, s'il n'aurait pas été préférable de laisser un seul blanc dans $\mathrm{T}$, correspondant au v. 2883 de L (où le rédacteur ajoute les noms de Roland et d'Olivier), et d'imprimer les trois vers suivants des deux versions en regard, ce qui aurait permis aussi de supprimer le blanc entre les v. 2886 et 2887 de L. C'est ce qui arrive à d'autres endroits, par ex. L 407, 444 vs T 245, 263 mentionnés plus loin.

La ponctuation du passage dans les deux versions soulève aussi un problème intéressant. Les éditeurs mettent une virgule à la fin de L v. 2882, et le v. correspondant de T $(2515$ - il faut noter que les blancs ne comptent pas dans la numérotation des textes), un point et virgule à la fin du v. 2883 de L et à la fin du v. 2516 de T, ce qui fait que du point de vue syntaxique et sémantique c'est ce vers ( «Et tous vos compaignons de ce palais volu ») qui remplace l'allusion à Roland et Olivier de L, allusion qui semble plus logique dans un contexte où l'auteur nomme Charlemagne. D'ailleurs dans L la construction négative se poursuit, tandis que dans $\mathrm{T}$, suivant l'édition de F. E. Denis et W. W. Kibler, le négatif est remplacé par un positif. En plus la présence du point et virgule à la fin de T 2516 rend le v. 2517 « orphelin »-puisque le verbe « seront » n'a pas de sujet exprimé dans le texte imprimé. Ceci n'est pas impossible en ancien français, mais le suivi du sens rendrait préférable l'insertion d'un point et virgule à la fin de T 2515 et de supprimer la ponctuation à la fin de T 2516 pour rétablir le lien entre l'image des compagnons et la pendaison de ceux-là. 
Si j'ai analysé si longuement ce passage, c'est que le type de problèmes qu'il soulève paraît ailleurs, mais en général les objections qu'on pourrait faire au travail des éditeurs sont très rares dans le reste de l'édition. On ne relève que « cil »= le Christ, sans majuscule (L 149) mais avec majuscule (T 161, qui y correspond) et inversement « Celui » (L 1279) contre « celui » (T 937); L 407 «E si fetes viande a dis ans mener » est imprimé face à face avec T 245 « S'irons après les peres qui nos ont engendrez », bien qu'il n'y ait pas d'équivalence entre ces deux vers; de même L 444 «E li enfant si firent checun en son pais » est imprimé en regard de T 263 «Et si i met vitaille a .x. ans acomplis ", qui fait écho à L 407, sans qu'un blanc soit inséré à l'un ou l'autre endroit. C'est sans doute par mégarde qu'on trouve un grand blanc dans L (l'équivalent de quatre vers entre 819 et 820 ) et dans T (au même endroit, l'équivalent de cinq vers entre 679 et 680); une virgule est déplacée L 2240 : "Les escuz devant els tienent, les branz d'acer» lire « Les escuz devant els, [...]»; L 3901 « L 4160 la correction « Cestu[i] » n'est pas nécessaire : la forme « cestu » est enregistrée par l'Anglo-Norman Dictionary, s.v. « cest »; L 4339, T 3988 ajouter une virgule après « fesant ».

L'Introduction commence par une description sommaire des éditions antérieures à celle de F. E. Denis et W. W. Kibler, mais deux de ces éditions, toutes deux du manuscrit de Tours, sont mentionnées pour la première fois, p. 11, au cours de la justification et la description de la nouvelle édition. Il s'agit de celle de Edward Guillén Brown Jr. (The Tours Manuscript of Gui de Bourgogne: An Annotated Edition, thèse de doctorat, University of Arizona, 1968, en ligne, URL : https://repository.arizona.edu/ handle/10150/284961 [consulté le 05/11/2021]) et celle d'Hélène Latour (Gui de Bourgogne, chanson de geste : édition critique, thèse de Diplôme d'archiviste paléographe, Paris, École nationale des chartes, 1976); ces deux éditions sont inédites. Au même endroit est mentionnée l'édition du fragment de Darmstadt qu'Irene Hall a ajoutée à son édition du manuscrit de Londres (Gui de Bourgoine, chanson de geste: First Critical Edition of the Manuscript Harley 527 [fols. $\left.1 r^{\circ}-32 r^{\circ}\right]$ of the British Museum, thèse de doctorat, University of Hull, 1962), mentionnée pour la première fois p. 9. Cette section est suivie par la description détaillée des deux manuscrits complets et des deux fragments. Le ms. Harley est une anthologie dont les deux éléments principaux sont Gui de Bourgogne et Le Roman de Horn anglonormand. Les éditeurs de Gui qualifient le texte de Londres comme étant « extrêmement négligé dans une langue de coloration anglo-normande » (p. 15), mais indiquent aussi que ce texte "négligé " a évidemment été relu soigneusement par le scribe et le chef de l'atelier, puisqu'on y remarque « de nombreuses corrections $»$ : cette distinction entre une apparence négligée et le soin mis à la préparation du texte n'est pas insolite dans les manuscrits anglo-normands d'œuvres vulgaires. En plus ils traitent la versification du texte de Londres comme étant " d'une irrégularité métrique presque invraisemblable » (p. 11), ce qui laisse supposer qu'ils la jugent selon les critères de la versification continentale. Pourtant, on sait depuis la communication de Dafydd Evans au congrès Rencesvals de Strasbourg, «La versification anglo-normande " (Au carrefour des routes d'Europe : la chanson de geste, $\mathrm{X}^{\mathrm{e}}$ congrès international de la Société Rencesvals pour l'études des épopées romanes [Strasbourg, 1985], Aix-enProvence, Université de Provence [Senefiance, 20-21], 1987, p. 473-488) l'importance de l'ictus plutôt que le simple compte syllabique dans la détermination de la longueur des vers. Aussi peut-on croire, d'après les nombreuses distinctions entre le texte Harley et celui de Tours, que nous avons affaire non pas à une simple copie d'un texte continental mais à une réécriture à l'intention d'un public insulaire. On note que bon nombre des vers ajoutés par le scribe anglo-normand offrent une explication plus claire et syntaxiquement plus logique que la parataxe de la version continentale, ce qui laisse deviner l'intervention de ce que Jeanne Wathelet-Willem a appelé « un scribe glossateur ». À titre d'exemple on peut citer les vers L 2887-2894 :

Quant l'entent Gui, si fu mult irascus.

E l'erceveske s'escrie : - « Sire Gui, que fes tu?

Menbre tei de ta mère ke dist le salu. »

E quant l'entent Gui, le cuer est esmu,

U k'il veit le paen li est coru.

L'enfes Gui de Burgoine forment s'aira

Quant oit cels de la tur ke chescun s'escria

Ke de sa mère li menbre, sa vertu recovra;

contre T 2518-2522

Quant l'enfes Guis l'entent, s'ot le cuer irascu;

Ou qu'il voit le paien, sore li est coru.

L'enfes Guis de Borgoigne durement s'aïra.

Ceus de la tour oï, dont chascuns s'escria ;

De sa mère li menbre, sa vertu recovra.

Dans la section dédiée à "L'État des recherches » les éditeurs passent en revue les opinions des savants publiées depuis le milieu du XIX ${ }^{\mathrm{e}} \mathrm{s}$. sur les rapports entre le poème, l'histoire et la géographie. Ils concluent, très pertinemment, que « la chanson refuse 
de se laisser enfermer dans des cadres historiques ou géographiques précis » (p. 41). Viennent ensuite la présentation de l'établissement des textes de tous les manuscrits (p. 56-66) et une étude de la langue des deux manuscrits principaux (p. 66-86). On regrette qu'ils n'aient pas ajouté une étude de la langue des fragments, surtout de Darmstadt qui offre un nombre de traits lorrains. Dans l'étude de la langue de L le manque de distinction entre [n] et [ñ] et entre [1] et [lj] est mis sur le compte du système graphique du scribe (p. 69), mais il s'agit proprement d'un trait phonétique du français insulaire (Mildred K. PoPe, From Latin to Modern French with Especial Consideration of AngloNorman: Phonology and Morphology, Manchester, Manchester University Press [Publications of the University of Manchester, 229], 1934, § 1182). Dans $\mathrm{T}$ ils indiquent que « -s est le signe ordinaire du pluriel » (p. 78), mais dans la liste des formes répertoriées ils incluent « cors » (indéclinable) et « cuens » (sujet singulier au v. T 39). L'édition est complétée par des notes pour la plupart critiques qui indiquent les corrections apportées aux textes, un glossaire et un index des noms propres. Le glossaire n'est pas exhaustif, et on note quelques formes et expressions que les éditeurs passent sous silence. Parmi celles-ci on remarque dans $\mathrm{L}$ 《 espees 》 = « espiez » (épieu, lance : L 2648), «manier » = « mener » (L 105) et la forme du $1 \mathrm{pl}$. " menamums »= " menâmes " (L 2490) qui ne figurent ni au glossaire ni dans l'étude de la langue. En revanche, « arcage » (T 430) est glosé « langage »; or, il est vrai que le mot figure dans le dictionnaire de Godefroy et dans le ToblerLommatzsch, mais ils ne citent que cet exemple, et Tobler-Lommatzsch ajoute un point d'interrogation après la glose. En fait on peut croire à une simple faute de copie dans un ancêtre de T qu'on aurait pu corriger, d'autant plus que le vers correspondant de $\mathrm{L}$, réduit à un octosyllabe, semble indiquer que son copiste ou un prédécesseur de celui-ci n'a pas compris la forme et l'a sautée. Une autre expression qui n'est expliquée nulle part est « nune de jur » (L 3845), « none de jor » (T 3487) : les exemples cités dans le Complément de Godefroy et dans le Tobler-Lommatzsch indiquent assez clairement qu'elle signifie " midi », comme « nune » tout court en anglo-normand (L 524), où le vers correspondant de T (308) a la leçon « midi »; puisqu'il s'agit dans ce passage d'une allusion à la mort du Christ selon les Évangiles synoptiques la glose « midi » convient parfaitement à la leçon de L. Somme toute, on constate que F. E. Denis et W. W. Kibler ont entrepris un projet important et complexe. Ils ont à la fin réussi à présenter aux étudiants et chercheurs une édition très utile de tous les témoins d'une chanson de geste intéressante et jusqu'ici méconnue.

Philip E. BenNeTt Université d'Édimbourg 